\section{Abstract 01}

DOI: $10.5935 / 2525-5711 . s u p 11.2020 .01$

\section{Oral surgical management in a patient with a history of breast carcinoma}

André Pereira Falcão, Lucyene Miguita, Juliana Mota Siqueira, Maria de Fátima Santos Meireles, Jeferson Ribeiro Máximo, Camila de Barros Gallo, Carina Domaneschi ${ }^{(1 D}$

Department of Stomatology, School of Dentistry, University of São Paulo, São Paulo-SP, Brazil

Surgical dental treatment for cancer patients requires more alertness in propaedeutics when in comparison to normoreactive subjects. Therapy must always be appropriate to the individual's clinical and systemic condition. Under those circumstances, we report a female patient, 51 years old, leucoderma, with a previous history of breast carcinoma. She underwent surgical excision, radiotherapy, and reconstruction of the right breast. The patient described experiencing pain around the 14 tooth area. Clinical and radiographical observations revealed the presence of a protuberance in the vestibule-distal region and in the alveolar ridge of the tooth. The protuberance is related to the local anatomy and previous exodontia, which occurred due to extensive coronary loss, deflection of the remaining cusps, presence of gingival hyperplasia covering the crown, endodontic treatment with the absence of gutta-percha in the region of the pulp chamber, and ankylosis. In her medication history, there was no use of anti-resorptive drugs, which would represent a surgical contraindication. The surgical planning for the region of the 14 tooth included flap making and vestibular and bone spike osteoplasty, with beveling of the osteotomy margins. An antibiotic was prescribed for 7 days and the patient should start taking it 2 days before the surgery. The subject had good post-surgical tissue repair and probable posterior prosthetic rehabilitation. Therefore, it is fair to conclude that careful anamnesis sets up an adequate surgical propaedeutics with a good prognosis, which may improve the quality of life of cancer patients.

\section{Abstract 02}

DOI: 10.5935/2525-5711.supl1.2020.02

\section{Dental management prior to oncological treatment in a rare and extensive case of osteosarcoma in the mandible}

Daniele Heguedusch; Marcus Vinícius Bueno; Camilla Vieira Esteves; Marilia Trierveiler; Celso Augusto Lemos Junior; Carina Domaneschi, Camila de Barros Gallo (D)

Department of Stomatology, School of Dentistry, University of São Paulo, São Paulo-SP, Brazil

A 59-year-old black female patient went to ambulatory care complaining about a painless swelling in the jaw that persisted for the last 20 days. During the extra-oral examination, a volume increase in the region of the right mandible was perceived. The intraoral examination found a nodule with a smooth surface and solid consistency in the region of the lower left third molar. Radiographic images show a poorly delimited mixed area of the region with a "sunburst" appearance. An incisional biopsy was performed, and the histopathological examination revealed mesenchymal neoplasia with a proliferation of spindle cells and large ovoid with small septa of organized osteoid, which was compatible with osteosarcoma. After the diagnosis, a dental appointment previously the oncological treatment was performed and the condemned dental elements $32,33,34,43,44$, and 45 were extracted in order to avoid infections during medical care. Osteosarcoma is a malignant lesion of mesenchymal cells in gnathic bones. It represents 6 to $8 \%$ of all lesions in the jaws and it occurs mainly between the third and fourth decades of life. The dental treatment during cancer care aims to remove the foci of infection that may adversely affect the continuation of cancer treatment and the patient's quality of life.

\section{Abstract 03}

DOI: $10.5935 / 2525-5711$. supl1.2020.03

\section{The use of low-power laser in the treatment of oral mucositis: a case report}

Isabela Américo Eutran da Silva, Flavia Mota de Souza, Gabriel Araújo da Silva, Karin Sá Fernandes

School of Dentistry, City University of São Paulo

Radiotherapy and/or chemotherapy leads to multiple oral complications, such as oral mucositis (OM), bacterial, viral, and fungal infections, loss of taste, hyposalivation, and trismus. OM is characterized by generalized inflammation in the oral mucosa amidst erythema, edema, whitish plaques, and ulcers with intense painful symptoms, which can cause a secondary infection. The World Health Organization (WHO) grades $\mathrm{OM}$ in a scale system that varies from $0,1,2,3$, and 4 , according to clinical characteristics and symptoms. The treatment consists of antibacterial mouthwashes, rigorous oral hygiene, topical 
anesthetics, powerful analgesics, and low-power laser. Laser therapy promotes cell proliferation and stimulates tissue healing, besides providing analgesia without side effects. Thus, the aim of this study is to report the clinical case of a 62-year-old female patient diagnosed with papillary thyroid carcinoma, plus metastasis in bones, lymph nodes, and adrenal gland. The patient underwent a total thyroid resection. Afterward, 2 sessions of iodine therapy, oral chemotherapy with Sorafenib for 8 months, and 20 sessions of 3D-RCT conformational radiotherapy with a dosage of $3 \mathrm{~Gy}$ in the scapular region, spine, and the femur. On the 12th day of chemotherapy, the patient presented erythema and whitish plaques in the mouth with mild painful symptoms. In a few days, it evolved to ulcers in the entire oral cavity and oropharynx region, meaning OM grade 4 (WHO). Under those circumstances, the use of the low-power diode laser $(660 \mathrm{~nm}$ and $808 \mathrm{~nm} ; 100 \mathrm{~mW}$; $2 \mathrm{~J} / \mathrm{cm} 2$ ) began, for a total of 24 sessions, on alternate days, with partial remission of the lesions and significant improvement in painful symptoms. Laser therapy has been very efficient for $\mathrm{OM}$, as it provides a significant improvement in pain and healing of oral lesions, thus, it brings a better quality of life for the patient.

\section{Abstract 04}

DOI: $10.5935 / 2525-5711 . s u p l 1.2020 .04$

\section{Dental approach to a patient with oncological sequelae: a clinical case report}

\section{Larissa Fernanda dos Santos Lima Macedo, Luara Maria Cerioni Gonçalves, Marina Paraluppi, Juliana Mota Siqueira, Camila de Barros Gallo, Carina Domaneschi}

Department of Stomatology, School of Dentistry, University of São Paulo, São Paulo-SP, Brazil

Head and neck squamous cell carcinoma is the fifth most common type of cancer worldwide. Its treatment is related to great morbidity due to possible sequelae, such as partial or total glossectomy and tracheostomy, as well as tube feeding. Considering the intensity of these treatments, whether surgical, chemo, and/or radiotherapy, several oral manifestations may occur and affect the individuals, significantly compromising their quality of life. All aspects must be taken into account for proper dental management of these patients. The objective of this study is to report the case of a 73 yearsold white female patient. She went to the clinic of the Interdisciplinary Academic League of Oral Cancer to adapt the pre-radiotherapy oral environment due to the diagnosis of malignant lesions in the oropharynx and rectum, with a history of alcoholism and smoking. She also had a previous history of oropharyngeal squamous cell carcinoma (2001), with subsequent partial glossectomy, tracheostomy, neck dissection, and radiotherapy. She used a nasogastric tube and antiplatelet medications, antidepressants, antihypertensives, and morphine. Clinical routines were followed to avoid discomfort and risks in view of the sequelae generated by previous cancer treatment. Restorative treatment, periodontal treatment, and oral hygiene instructions were performed. In the literature, there was no specific protocol to approach similar cases. Thus, as the number of people affected by such dysfunctions is relevant, it is necessary to develop a routine to outline the dental care for these individuals in a secure way for both the patient and the health care professionals.

\section{Abstract 05}

DOI: $10.5935 / 2525-5711 . s u p l 1.2020 .05$

\section{Importance of oral supportive care after head and neck radiotherapy: a case report}

\author{
Marcus Vinícius Bueno, Daniele Heguedusch, Tamires Feliciano \\ Floriano, Giovanna Nogueira Pareja, Samanta Vicente de
} Oliveira, Carina Domaneschi, Camila de Barros Gallo

Department of Stomatology, School of Dentistry, University of São Paulo, São Paulo-SP, Brazil

A 74-year-old black female patient was diagnosed and treated for squamous cell carcinoma (SCC) in the left hard palate region by surgery, chemo, and radiotherapy. She was referred to outpatient care for oral rehabilitation. Intraoral examination revealed a nasopalatine communication on the left side, a sequel to oncological surgical treatment, and the absence of several dental elements. Of the present teeth, only the 17 was healthy. The others $(16,45$, $43,42,41,31,32,33$, and 34) exhibited radiation caries in need of restorative, endodontic, and surgical treatment. Dental extractions implicated the risk of developing osteoradionecrosis, so the surgical procedures of the elements that were not suitable for rehabilitation were performed one at a time, with antibiotics prophylaxis and therapy, and with special care to improve bone and tissue healing, like minimum trauma, osteoplasty and occlusive suture. It was also performed photobiomodulation to control post-op edema $(830 \mathrm{~nm}, 70 \mathrm{~mW}$, 2J per point, and $29 \mathrm{~s}$ ) and cleaning of the surgical area with $0.12 \%$ chlorhexidine digluconate. The management of cancer patients after radiotherapy must be as conservative as possible. Avoiding major complications is vital, such as osteoradionecrosis of the jaws, a deleterious condition of radiotherapy. Also, it is crucial to provide the patient with the best treatment in view of their prosthetic rehabilitation. Osteoradionecrosis is one of the most severe complications in patients undergoing radiotherapy 
treatment for head and neck cancer. It means ischemic bone necrosis due to low vascularization and osteoclastic activity, which affects bone metabolism and remodeling, possibly causing pathological fractures.

\section{Abstract 06}

DOI: $10.5935 / 2525-5711 . s u p l 1.2020 .06$

\section{Dental care in a patient with advanced oral cancer under palliative treatment}

Tamires Feliciano Floriano, Daniele Heguedusch, Giovanna Nogueira Pareja, Renata dos Santos Vellei, Samanta Vicente de Oliveira, Carina Domaneschi, Camila de Barros Gallo

Department of Stomatology, School of Dentistry, University of São Paulo, São Paulo-SP, Brazil

A 52-year-old white male patient attended the clinic complaining about mandibular teeth mobility. During anamnesis, he reported radiotherapy for tongue squamous cell carcinoma (TSCC) 5 years ago. He stated that he developed osteoradionecrosis (ORN) due to tooth extraction 3 years ago. The intraoral examination revealed that the ORN disturbed the anterior region with extension to the jaw body on the right side and that there was a recurrence of the TSCC on the left side. Panoramic radiography showed two pathological mandibular fractures due to ORN and TSCC. The patient was undergoing palliative chemotherapy and in control of his general health. Dental care consultations were held weekly. The patient underwent biofilm control with chlorhexidine irrigation and photodynamic therapy, sequestrectomies, and extractions. In addition, the subject asked for aesthetic dental restorations and adjustments of the removable partial denture. Palliative treatment corresponds to health care for patients whose disease does not respond to curative treatment. It manages the patient's pain and psychosocial factors, besides supporting their family members. The understanding of the individual as a social being, with values and beliefs, contributes to improving the quality of life in the terminal phase of life.

\section{Abstract 07}

DOI: $10.5935 / 2525-5711 . s u p 11.2020 .07$

\section{Removal of veneers with Er, Cr: YSGG laser after in vitro radiotherapy simulation of head and neck}

Thais Freitas Rabelo, Claudia Bianchi Zamataro, Nathalia Zanini, Amanda Caramel Juvino, Nielsen Grosko Kuchar, Pedro Castro, Denise Zezell ${ }^{\circledR}$

Lasers and Applications Center - Institute of Energy and Nuclear Research - IPEN - CNEN / SP

Contemporary dentistry is characterized by the search for aesthetic perfection of the smile. For this reason, common people are frequently demanding porcelain veneers and dental contact lenses so they can achieve the ideal of formal perfection dreamed by the Greeks since ancient times. Ceramic laminates, when correctly employed, can bring numerous psychosocial benefits to the patient. However, it is worrying that a great number of young people pursuit these procedures exclusively for aesthetics reasons. Restorative materials do not last for life and these patients are more susceptible to facing health problems, such as head and neck cancer, whose radiotherapy treatment can make it difficult to remove old facets. Objective: To evaluate, in-vitro, the set of human dental enamel, laminated veneers of porcelain, and resin cement after ionizing (gamma radiation) and non-ionizing radiation (Er, Cr: YSGG laser). After CEP-FOUSP (CAEE 02717618.8.0000.0075) granted an approval, 20 samples of human dental enamel were used and divided into 2 groups $(\mathrm{n}=10)$ : negative control group (laser) and test group (gamma + laser). In the test group, we applied $0.07 \mathrm{kGy}$ of gamma radiation, which is the cumulative dose of ionizing radiation in the treatment of head and neck cancer. Afterward, the facets were cemented to the enamel with Variolink ${ }^{\circledR N}$ cement. Subsequently, the facets were removed by a laser, according to the protocol (3.5 W, $20 \mathrm{~Hz}, 175 \mathrm{~mJ}, 1296 \mathrm{~W} / \mathrm{cm} 2,64 \mathrm{~J} / \mathrm{cm} 2)$. SEM, EDS, IR spectroscopy, and mechanical shear tests were performed. The microscopic images obtained after laser irradiation in the negative control group preserved the original morphological characteristics of the enamel prisms in the form of a "lock," while in the test group, the prisms were organized in the form of "hives," due to water radiolysis. While analyzing the intra-group IR spectroscopy, the average of the spectra before and after laser facet removal did not show any significant difference. There was no formation of new compounds on the enamel surface, which the EDS analysis confirmed. Using Er, Cr: YSGG laser in the removal of laminated porcelain veneers after gamma radiation appears to be efficient. However, it is necessary to establish the intra-pulp dental temperature during laser irradiation to prevent iatrogenic and healthy dental structures. 